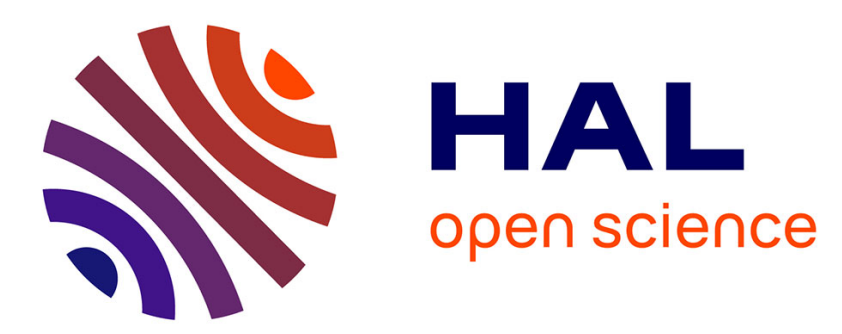

\title{
De la plage appropriée à la plage patrimonialisée, analyse de pratiques balnéaires "déviantes" sur le littoral camarguais: l'exemple de Piémanson et Beauduc
} Cécilia Claeys, Laurence Nicolas

\section{- To cite this version:}

Cécilia Claeys, Laurence Nicolas. De la plage appropriée à la plage patrimonialisée, analyse de pratiques balnéaires "déviantes" sur le littoral camarguais: l'exemple de Piémanson et Beauduc. Rives Méditerranéennes, 1999, Dynamiques naturelles et gestion des espaces littoraux., 93, pp.2734. 10.3406/medit.1999.3127 . hal-02483680

\section{HAL Id: hal-02483680 \\ https://hal.science/hal-02483680}

Submitted on 18 Feb 2020

HAL is a multi-disciplinary open access archive for the deposit and dissemination of scientific research documents, whether they are published or not. The documents may come from teaching and research institutions in France or abroad, or from public or private research centers.
L'archive ouverte pluridisciplinaire HAL, est destinée au dépôt et à la diffusion de documents scientifiques de niveau recherche, publiés ou non, émanant des établissements d'enseignement et de recherche français ou étrangers, des laboratoires publics ou privés. 


\title{
De la plage appropriée à la plage patrimonialisée, analyse de
} pratiques balnéaires «déviantes» sur le littoral camarguais : l'exemple de Piémanson et Beauduc

In: Méditerranée, Tome 93, 4-1999. Dynamiques naturelles et gestion des espaces littoraux. pp. 27-34.

\begin{abstract}
Within the Camargue area, a 30 kilometer coastal linear strip is used either temporarily by rough campers (Piémanson), or permanently by small house users (Beauduc). For the sake of the Maritime Public Domain (MPD), the State authorities wish to get rid of that clearly illegal occupancy. A sociological and ethnological approach shows the social and symbolical logics that underlie such usages of the coast and that illustrate a frequent conflict in environment between administrative standards and social habits.
\end{abstract}

\section{Résumé}

En Camargue, 30 kilomètres de linéaire côtier sont utilisés soit temporairement par des campeurs sauvages (Piémanson), soit en permanence par des cabaniers (Beauduc). Au nom du respect du Domaine public maritime (DPM), les autorités de l'État souhaitent voir disparaître ces occupations objectivement illégales. Une approche sociologique et ethnologique révèle les logiques sociales et symboliques qui sous- tendent de tels usages du littoral, illustrant un conflit, fréquent en environnement, entre normes administratives et habitudes sociales.

Citer ce document / Cite this document :

Claeys-Mekdade Cécilia, Nicolas Laurence. De la plage appropriée à la plage patrimonialisée, analyse de pratiques balnéaires «déviantes» sur le littoral camarguais : l'exemple de Piémanson et Beauduc. In: Méditerranée, Tome 93, 4-1999. Dynamiques naturelles et gestion des espaces littoraux. pp. 27-34.

doi : 10.3406/medit.1999.3127

http://www.persee.fr/web/revues/home/prescript/article/medit_0025-8296_1999_num_93_4_3127 


\title{
De la plage appropriée à la plage patrimonialisée,
}

\author{
analyse de pratiques balnéaires «déviantes» sur le
}

\section{littoral camarguais : l'exemple de Piémanson et Beauduc}

\begin{abstract}
Résumé - En Camargue, 30 kilomètres de linéaire côtier sont utilisés soit temporairement par des campeurs sauvages (Piémanson), soit en permanence par des cabaniers (Beauduc). Au nom du respect $d u$ Domaine public maritime (DPM), les autorités de l'État souhaitent voir disparaître ces occupations objectivement illégales. Une approche sociologique et ethnologique révèle les logiques sociales et symboliques qui soustendent de tels usages du littoral, illustrant un conflit, fréquent en environnement, entre normes administratives et habitudes sociales.
\end{abstract}

\author{
Cécilia CLAEYS-MEKDADE* \\ Laurence NICOLAS*
}

\section{1 - HISTORIQUE}

Depuis le milieu du $\mathrm{XX}^{\mathrm{e}}$ siècle, les vastes étendues sablonneuses du littoral camarguais exercent un fort pouvoir attractif auprès des populations locales. Ce littoral, éloigné des principales localités habitées, reste d'un accès difficile et aléatoire jusque dans les années 1950-1970. Sans desserte routière, il n'est jusque là fréquenté que par des populations exclusivement maritimes comme les pêcheurs, les douaniers, les agents de la santé ou les baliseurs' ${ }^{1}$. La seule commune littorale du delta rhodanien située au bord de la mer est celle des Saintes-Maries-de-laMer. C'est donc sur ses rivages que vont commencer à se développer les premiers usages et pratiques balnéaires. Dès les années 1930-1940, les habitants des hameaux et des mas environnants mais aussi les citadins d'Arles effectuent des séjours sur la plage des Saintes. Les campements sont alors rudimentaires : des bâches tendues, des chars à bœufs, des camions ou autres engins agricoles sont utilisés.

\begin{abstract}
Within the Camargue area, a 30 kilometer coastal linear strip is used either temporarily by rough campers (Piémanson), or permanently by small house users (Beauduc). For the sake of the Maritime Public Domain (MPD), the State authorities wish to get rid of that clearly illegal occupancy. A sociological and ethnological approach shows the social and symbolical logics that underlie such usages of the coast and that illustrate a frequent conflict in environment between administrative standards and social habits.
\end{abstract}

Les pêcheurs y campent aussi depuis longtemps ${ }^{2}$, lorsque par exemple ils effectuent des campagnes de pêche sur des lieux éloignés de leur port d'attache. D'autre part le pèlerinage lié à l'église des SaintesMaries draine chaque année une foule de fidèles qui n'hésitent pas à s'établir deux ou trois jours, ou seulement pour la journée, au bord de la mer. Au lendemain de la guerre, l'établissement estival de ces populations locales devient essentiellement balnéaire. Jusqu'en 1981, l'accès du littoral est libre et la pratique du camping sauvage s'y développe sur des kilomètres (PICON, 1985, 1987). À cette époque, la commune des Saintes-Maries-de-la-Mer met en place une politique d'aménagement touristique, avec l'interdiction du camping sauvage et le développement de différentes structures d'accueil, ainsi qu'une action de protection de son littoral et se soumet avant l'heure à la future loi Littoral de 1986.

\footnotetext{
* Laboratoire DESMID (Dynamique écologique et sociale en milieu deltaïque) - ESA CNRS 5023, Arles.
} 
Il en va tout autrement pour l'autre moitié du littoral camarguais. Situé sur la commune d'Arles dont il est distant d'une cinquantaine de kilomètres, le bord de mer arlésien est quant à lui d'un accès difficile, voire impraticable. La mise en place de I'exploitation salinière, au milieu du XIX ${ }^{\mathfrak{c}}$ siècle, dans cette partie sud-est du delta, a relégué au second plan les possibilités balnéaires ou de loisirs pouvant être liées à sa frange maritime. La mer est pourvoyeuse de sel pour l'industrie, c'est là sa vocation principale. Cependant l'installation en 1956 de la station de pompage, qui fournit l'eau de mer nécessaire à l'exploitation, à l'endroit du grau du
Galabert améliore considérablement l'accès de la partie ouest du littoral arlésien par la construction et l'entretien de pistes raccordées à l'ancienne digue à la $\mathbf{m e r}^{3}$. Dès lors, la pratique et la fréquentation de l'espace maritime lagunaire de Beauduc peuvent se développer. La partie est, plage de Piémanson ou plage d'Arles, n'est quant à elle reliée à la route D36 qu'à partir de 1971. Aujourd'hui seules les plages de Beauduc et de Piémanson échappent à la nouvelle législation, et se distinguent fortement du reste du littoral camarguais -et peut-être de l'ensemble du littoral français-par les pratiques, les usages et les représentations qui se rattachent à elles.

\section{2 - UNE APPROPRIATION SYMBOLIQUE ET MATÉRIELLE DE L'ESPACE}

Les usages ici étudiés ont en commun l'infraction à la loi Littoral. À Beauduc comme à Piémanson, durant la belle saison, des populations investissent le rivage selon des modalités comparables -bien que sensiblement différentes dans les deux cas- et que l'on peut caractériser comme une appropriation symbolique et matérielle d'un espace depuis longtemps défini comme espace public.

Dès 1681, l'ordonnance de Colbert assigne au rivage, «tout ce que la mer couvre et découvre, et jusqu' où le grand flot de mars se peut étendre sur les grèves», le statut de Domaine public maritime par définition inaliénable et imprescriptible. Celui-ci est ainsi délimité sur l'ensemble des côtes françaises à l'exception de celles de la Méditerranée (FLEURY, 1992) où en raison de l'absence des marées, mais pour ne pas réduire l'étendue du DPM s'applique une autre règle, issue du droit romain, «le rivage de la mer s'étend jusqu'à l'endroit où arrive le plus grand flot d'hiver» ${ }^{4}$, ces deux types de délimitation, dont l'une se réfère au plus grand flux de mars et l'autre au plus haut flot de l'hiver, sont maintenues jusqu'en 1973. En effet, l'arrêt KreitmanN du Conseil d'État du 12 octobre 1973 fixe désormais la limite du DPM «au point où les plus hautes mers peuvent s'étendre en l'absence de perturbations météorologiques exceptionnelles» et ce, quel que soit le rivage.

La loi Littoral du 3 janvier 1986 s'inscrit quant à elle dans une volonté politique de protection du littoral et de préservation des zones où s'observent encore des coupures d'urbanisation. Cette loi est promulguée en réaction au bétonnage massif des côtes françaises. Elle prévoit l'interdiction sur la bande littorale de constructions et d'installations, du camping-caravaning et de la circulation automobile.

Toute pratique d'autoconstruction, ou d'établissement balnéaire provisoire de type campingcaravaning est donc proscrite. Cependant, une dérogation à usage professionnel était traditionnellement accordée aux pêcheurs qui pouvaient édifier, sur les rivages, des cabanes destinées principalement à abriter les engins nécessaires aux pratiques halieutiques mais utilisées également comme habitat saisonnier. C'est sur ce modèle -en tant que modèle culturelque se développe l'habitat cabanier de Beauduc, même si, tout comme à Piémanson, ces installations saisonnières ou permanentes, s'inscrivent surtout dans une perspective de villégiature balnéaire.

\subsection{Spécificité des usages : le permanent et le temporaire}

Beauduc et Piémanson se différencient surtout par le mode d'occupation de l'espace. L'implantation beauducoise est permanente, dans la mesure où une partie des usagers ont construit des cabanons. Beauduc, site maritime lagunaire, a fait l'objet d'appropriations successives depuis sa formation au milieu du XVIII ${ }^{\mathrm{e}}$ siècle. Les premières formes d'appropriation de ce littoral commencent, nous l'avons $\mathrm{vu}$, avec une population maritime. Après la naissance de la cité industrielle de Salin-de-Giraud, ce sont ensuite des saliniers, ou des Arlésiens pêcheurs occasionnels, qui arpentent ce territoire lagunaire alors non endigué et véritable pays de cocagne pour la pêche «amateur» dans les étangs et les lônes ${ }^{5}$. À partir des années 1950, durant l'été, des familles d'origine locale, rurale ou urbaine, viennent y passer leurs vacances et se construisent à leur tour des cabanes. Cette population, socialement homogène, imprime bientôt au lieu une caractéristique culturelle et paysagère, donnant à ce bout de littoral, une allure pour le moins originale et singulière.

Aujourd'hui, 400 occupations en tout genre (cabanons, caravanes, autobus, abris de chantier, fourgons...) regroupées en 280 installations (correspondant à des unités familiales ou amicales) se répartissent sur une vaste étendue sableuse d'environ 60 hectares. Ces différentes unités d'habitats, 
saisonnières ou permanentes, ont peu à peu formé trois quartiers d'importance, Beauduc-nord, Beauduc-plage et Beauduc-village. Certains cabaniers y séjournent toute l'année (pêcheurs, restaurateurs, retraités...), d'autres sont des villégiateurs saisonniers. L'ensemble de cette population est venue s'installer sur les lieux dans une perspective liée au prélèvement sur la nature (pêche en mer et en étang, ramassage de coquillages). Motivés par le plaisir -sinon la passion- de la pêche, par le complément non négligeable que celle-ci peut représenter sur un plan alimentaire ou au niveau des ressources. Les nombreux adeptes de Beauduc sont également connus sous le sobriquet collectif de «gratte-plage». Leur rapport particulier à la nature explique et justifie un tel surnom : transformés durant la belle saison en véritables hommesfouisseurs, ils s'adonnent avec constance et délectation à la recherche de toutes sortes de coquillages ${ }^{6}$ que l'on peut trouver dans les faibles fonds sablolimoneux du golfe. Par extension ce surnom, dont ils s'affublent eux-mêmes avec humour et dérision, recouvre également tout autre activité liée au prélèvement (pêche plaisancière en mer ou en étang) ou à la récupération (objets ou matériaux pouvant améliorer leurs habitations de fortune). En plus de ces cabaniers, les périodes estivales attirent des campeurs et caravaniers, ainsi que des visiteurs à la journée. On note également depuis une dizaine d'années, l'apparition d'un nouveau groupe d'usagers, les véliplanchistes, présents sur le site tout au long de l'année, durant les week-ends et les périodes de vacances.

L'implantation piémansonnaise, en revanche, est exclusivement temporaire. Inoccupée l'hiver, la plage est uniquement fréquentée pendant cette période par des chasseurs et des pêcheurs (amateurs ou professionnels ${ }^{7}$ ). En période estivale, la plage est investie par les baigneurs à la journée, ainsi que par quelques milliers de campeurs et caravaniers. Les premiers campements sont installés dès le mois d'avril, les derniers à quitter la plage partent à la fin du mois de septembre. On y vient pour la baignade et le bronzage, ainsi que pour des loisirs tournés vers la sociabilité comme la pétanque. La pêche est aussi pratiquée par les plaisanciers, en mer avec des embarcations légères ou sur le rivage, pour le prélèvement de coquillages et la pêche à la ligne. Camper sur Piémanson est essentiellement une pratique familiale. Ces campements provisoires sont le lieu de regroupements familiaux, permettant à des familles géographiquement éclatées tout au long de l'année de se retrouver pendant l'été. Certains campements restent sur place les trois ou quatre mois de la belle saison, occupés en permanence par une ou deux personnes, retraité ou sans emploi. Le reste de la famille élargie (plusieurs générations et plusieurs fratries) s'y retrouve pendant les weekends et les congés de chacun ${ }^{8}$.

\subsection{Des usages socialement marqués}

L'émergence et le développement de cette forme d'usage du littoral camarguais s'inscrit dans un double processus historique. L'un concerne l'avènement des loisirs (CORBIN, 1995) l'autre touche plus particulièrement ce que $A$. CorbIN (1988) a désigné comme le désir du rivage.

Dès le milieu du $\mathrm{XIX}^{\mathrm{e}}$ siècle, la révolution industrielle entraîne un réaménagement des rythmes de travail, se caractérisant par une dissociation nette entre temps de travail et temps libre. Ces grands changements du XIX ${ }^{\mathrm{e}}$ siècle offrent un terrain favorable à la brusque mutation de l'aprèsguerre. La diffusion des congés payés, le possible accès aux vacances pour tous, permettent ce que J. Dumazedier (1988) désigne comme la révolution culturelle du temps libre.

La démocratisation des vacances prend toute son ampleur sur la plage. L'attrait du rivage n'a cependant pas toujours été chose évidente. Longtemps représenté comme espace d'insécurité physique et sociale, le littoral était associé à l'insalubrité et à la marginalité. $\mathrm{Au} \mathrm{XIX}^{\mathrm{e}}$ siècle, la première conquête du rivage est médicalisée. Il faut attendre le siècle suivant pour que l'usage du rivage devienne hédoniste. Aux bains froids et au suivi médical des établissements luxueux de thalassothérapie, se substitue l'attrait du sable chaud, du soleil et des bains de mer estivaux. Progressivement, la plage n'est plus le monopole des seules classes privilégiées. Les pratiques de plage n'en restent pas moins socialement et géographiquement différenciées (UrBAIN, 1994). Il va sans dire que la résidence tropézienne pied dans l'eau, n'a que peu à voir avec le studio loué sur la côte languedocienne, et moins encore avec le cabanon marseillais. Le territoire du vide (Corbin, 1988) des siècles passés est devenu un objet particulièrement convoité, les différentes classes sociales s'appropriant symboliquement et/ ou matériellement partie ou tout du littoral.

Les usages du littoral camarguais étudiés ici s'inscrivent donc dans cette double évolution du temps libre et du désir de rivage. Cette villégiature temporaire ou permanente est essentiellement une pratique populaire9. Les cabanons construits et les caravanes installées «sans droit ni titre» constituent une appropriation du site d'abord symbolique.

\subsection{Une appropriation symbolique : construction d'une mémoire collective}

$\mathrm{Au}$ lendemain de la guerre se développe l'usage et la fréquentation de cette partie du littoral arlésien. À Beauduc, en particulier, s'édifie chaque année un certain nombre de cabanes aux côtés de celles des pêcheurs ${ }^{10}$. Seuls ces derniers y vivent à 
l'année, pêcheurs en mer, pêcheurs de tellines (pêcheurs à pied) ou pêcheurs d'étang. Ils sont véritablement les figures pionnières qui ont investi le site. Pour la plupart aujourd'hui disparus ou repartis s'installer ailleurs, ces pêcheurs constituent les ancêtres fondateurs, le «panthéon» sur lequel se fonde la mémoire du lieu. Avec eux apparaissent les différentes formes qu'adopte bientôt la villégiature balnéaire : un habitat constitué et composé à partir de matériaux de récupération. La présence permanente des pêcheurs leur confère d'emblée un statut privilégié au sein de cette microsociété en formation, et c'est cette figure centrale, originelle et dominante du pêcheur qui lui donne un profil particulier. Cette microsociété se caractérise par une commune appartenance spatiale ; un ensemble de pratiques régit en effet l'exploitation de ce territoire et son accès est largement sanctionné par l'appartenance au groupe et la maîtrise symbolique particulière de cet espace (connaissance et désignation des lieux). Elle s'appuie aussi sur un espace de relations sociales, de liens sociaux (rapports de parenté, relation de coopération...) qui ont guidé par exemple les logiques d'implantation. Elle a également donné lieu à un parler local ou tout au moins en partie codé. Cette commune appartenance historique (mémoire locale) a scellé l'unité du groupe à travers un ensemble d'événements et leurs retombées locales (constructions de digues, inondations, procès...) et a abouti à l'existence d'une forme de pouvoir local représenté par le groupe des pêcheurs et des anciens cabaniers.

L'histoire de Piémanson est plus dépouillée que celle de Beauduc, elle est aussi moins ancienne. L'inscription spatiale des cabanons beauducois permet une lecture de l'histoire du site, que l'occupation temporaire de Piémanson n'autorise pas. Piémanson n'a pas ses familles de pêcheurs installées depuis plusieurs années. Si la pratique de la pêche y est certainement ancienne, si elle tient encore une place de choix dans les activités des villégiateurs, elle ne connaît cependant pas d'événements marquant, comme l'installation des premiers cabanons beauducois. Trois grands événements marquent néanmoins les mémoires des Piémansonnais. Le premier est l'achèvement, en 1971 , de la route entre le village de Salin-de-Giraud et la plage de Piémanson. L'ancienne piste est goudronnée, permettant un accès plus facile. Le second est l'interdiction, en 1981, du camping sauvage sur la plage des Saintes-Maries. Suite à cette restriction, le nombre des villégiateurs aurait augmenté, dans la mesure où les anciens des Saintes-Maries auraient trouvé refuge à Piémanson. Le troisième événement marquant la mémoire du site est la vague déferlante de 1985. Cette vague a opéré une sélection parmi les villégiateurs, à Piémanson comme à Beauduc. Certains ne sont plus jamais revenus, tandis que pour les autres, cette vague a été l'occasion de renforcer leur attachement à chacun de ces sites, en montrant leur capacité à gérer l'événement dans la solidarité et en acceptant l'inconfort et les risques liés à leurs pratiques.

La référence à ces événements passés contribue à l'élaboration d'une mémoire collective propre aux villégiateurs beauducois et piémansonnais. La production et la transmission de cette mémoire collective participe également à la construction identitaire du groupe.

\section{3 - SOCIÉTÉS ILLÉGITIMES ET SOCIÉTÉS HORS NORME : UNE CONSTRUCTION IDENTITAIRE}

\subsection{Des représentations ${ }^{12}$ et des pratiques partagées}

Ces villégiateurs beauducois et piémansonnais ont en commun une certaine représentation du site. Les données quantitatives recueillies auprès des usagers de Piémanson viennent conforter l'analyse qualitative du discours des Beauducois. Le discours recueilli a été regroupé en six catégories : la liberté (26\% des réponses), la convivialité et le bien-être $(23 \%)$, le balnéaire $(17 \%)$, la nature $(16 \%)$, et l'espace et la place (12\%)

Le thème le plus souvent évoqué est donc la liberté. Cette notion de liberté est large, allant de «pouvoir venir avec le chien», jusqu'à «ne pas être esclave de la société». Ces différentes références à la liberté ont en commun l'absence de contraintes ou plus exactement le choix de certaines contraintes pour échapper à d'autres. En contrepartie d'un confort sommaire et de contraintes matérielles (pas de téléphone, pas d'eau courante, pas d'électricité...), les villégiateurs trouvent à Piémanson et à Beauduc une absence de contrainte sociale, que chacun exprime à sa façon : «on s'installe comme on veut, on fait ce qu'on veut", "personne nous dit ce qu' on a à faire», «absence de servitude»...

Le thème de la convivialité et du bien être arrive en second. La convivialité est un sentiment fort chez les Beauducois et les Piémansonnais. Il renvoie à des pratiques essentiellement familiales et amicales. Le bon voisinage et la solidarité sont des attitudes valorisées.

Les références au balnéaire rappellent que les pratiques étudiées s'inscrivent dans un mouvement plus large, le désir de rivage. Venir à Piémanson ou à Beauduc, c'est aussi chercher une place au soleil, 
jouir de la mer et du sable chaud. Le «gratte-plage» étudié ici n'a certes que peu à voir avec le baigneur mondain de la côte d'Azur, il n'en reste pas moins qu'ils ont en commun une représentation hédoniste du littoral. Ils se différencient par leur définition de l'hédonisme.

Le thème de la nature est aussi mobilisé. Cette nature-là est éminemment sauvage, elle renvoie au thème de la liberté et à une symbiose primaire entre l'homme et les ressources naturelles. Ici, pas de panneaux indiquant le chemin à suivre, pas même de chemin, pas de pancartes donnant le nom des plantes en latin. Sont absentes donc la pancarte et la violence symbolique des noms en latin signifiant un regard et des pratiques de nature intellectualisées, propres aux couches sociales culturellement dominantes. Parce que sans repères culturels présupposés, cette nature sauvage est représentée par les villégiateurs comme échappant au contrôle de la civilisation. La civilisation, c'est le béton, la ville, mais aussi la hiérarchie et le contrôle social.

Le thème de l'espace vient renforcer cette idée. C'est un espace sans contrainte, où le villégiateur peut $s^{\prime}$ installer où bon lui semble, sur un site qu'il perçoit comme étant sans limite et sans délimitation physique, symbole d'une apparente absence de contrôle social. C'est aussi un espace où il y a de la place, où l'«on n'est pas les uns sur les autres ", soit parce qu' on a choisi de s'installer loin de la foule, soit parce que la promiscuité qui peut caractériser certaines zones est une promiscuité choisie et non pas imposée, une promiscuité recherchée et une convivialité, avec les membres de la famille élargie et les amis.

\subsection{Le refus de la norme et l'auto- organisation}

Les différents éléments de la représentation des Beauducois et des Piémansonnais s'articulent entre-eux. Ce désir de liberté, de nature sauvage, de larges espaces et de convivialité ont en commun la recherche d'un mode de vie différent, et plus largement d'un refus de la norme ${ }^{13}$. En voulant échapper au contrôle et à la hiérarchie sociale, ces individus ne prônent pas pour autant l'anarchie ou la révolution. Cette recherche de liberté et d'espace sauvage s'inscrit dans la production d'une auto-organisation ou autogestion. Ainsi, l'opposition à la norme s'articule à la formulation d'une autre norme, qui n'est plus imposée de l'extérieur, mais produite par le groupe lui-même. Ce ne sont pas les principes d'ordre et d'organisation qui sont rejetés, mais le fait qu'ils soient imposés de l'extérieur, produits par des groupes sociaux culturellement dominants, véhiculant des représentations et des pratiques dans lesquelles les couches populaires présentes sur Beauduc et Piémanson ne se retrouvent pas. Ce désir d'autoorganisation est explicitement exprimé par les Beauducois et les Piémansonnais : «on fait ce qu'on veut, on se débrouille, on demande rien à personne», «la liberté en respectant le lieu et les gens», «les gens s'organisent comme ils veulent, ils sont autoorganisés». Ces revendications d'autogestion rendent compte de l'étroit rapport entre norme et anti norme, montrant comment des groupes désignés comme déviants (BECKER, 1985), comme hors norme, produisent leurs propres règles, leurs propres normes. Ces représentations communes et ces revendications d'autogestion renforcent l'identité du groupe des Beauducois et des Piémansonnais.

\section{4 - SOCIÉTÉS ILLÉGITIMES FACE AUX REPRÉSENTANTS DE LA LOI : LE VILLÉGIATEUR, LE PRÉFET ET LE SCIENTIFIQUE}

\subsection{Les points de conflits et leur inscription sociale}

Bien qu'en infraction face à la loi, la villégiature piémansonnaise et beauducoise a longtemps été tolérée par les pouvoirs publics. Après cette période de tolérance, des campagnes d'information sont lancées, jouant le rôle d'un premier avertissement. Pour Beauduc, les premières procédures juridiques se concrétisent à partir de 1995. Concernant Piémanson, la mise en place d'un enrochement devant limiter l'accès aux véhicules débute en décembre 1995. Dans les deux cas, l'État désire reconquérir son domaine public maritime, en éradiquant ces pratiques de villégiature au nom de la sécurité, de l'hygiène et de la protection de l'environnement. En réponse à ces premières mesures, les villégiateurs ont organisé plusieurs manifestations et ont sollicité des rencontres avec les représentants des pouvoirs publics (mairie et sous-préfecture).

L'État se soucie de ses responsabilités civiles, souhaitant que les règles d'hygiène et de sécurité définies par la loi soient respectées sur l'ensemble de son territoire. De même, et plus récemment prises en compte, la protection de l'environnement fait aussi partie de ses préoccupation ${ }^{14}$. Comme le souci étatique concernant l'hygiène fut étroitement lié aux progrès de la médecine (CORBIN, 1982), de même le souci pour la protection de l'environnement est étroitement lié au développement de l'écologie scientifique (FABIANI, 1985). Les représentants de l'État et les scientifiques véhiculeraient des représentations de la nature spécifiques, largement partagées par les couches sociales culturellement dominantes ${ }^{15}$. 
Cette analyse permet de ne plus considérer le conflit comme la seule opposition entre norme et hors norme, mais d'en saisir plus précisément le contexte social et culturel. Au-delà d'une opposition entre représentants de la loi et sociétés illégitimes, ces divergences rendent compte de conflits plus larges. Ainsi, ces conflits d'usage peuvent s'interpréter comme des conflits de représentations de la nature et du littoral. Il s'agit d'une mésintelligence entre des représentations populaires et les représentations des couches sociales culturellement dominantes. Nous avons vu précédemment quelles sont les représentations des villégiateurs beauducois et piémansonnais (absence de contraintes, convivialité, pratiques de prélèvements). Elles correspondent largement aux représentations populaires de la nature mises en évidence par de précédents travaux (Picon, 1987 ; Chamboredon, 1985). Les représentations des couches moyennes intellectuelles se caractérisent, quant à elles, par un souci de patrimonialisation de la nature au nom des générations futures et par un rapport à la nature intellectualisé, à visée contemplative et d'apprentissage culturel (Kalaora, 1993 ; Aspe 1992 ; Picon, 1988 ; Chamboredon, 1985).

\subsection{La cohésion du groupe face à l'«autre»}

Beauducois et Piémansonnais se sont mobilisés face à la remise en cause de leur mode de fréquentation du littoral. Cette mobilisation est essentiellement associative, elle s'appuie sur des associations anciennes, mais aussi sur d'autres constituées suite aux premières actions de l'État. L'existence de plusieurs associations rend compte des différentes pratiques des villégiateurs (cabaniers versus caravaniers, naturistes versus «textiles»). Ces différentes associations se sont à plusieurs reprises mobilisées ensemble pour faire entendre leur voix. Au-delà de cette mobilisation associative, les décisions étatiques ont eu pour conséquence une cohésion accrue du groupe ${ }^{16}$. Se retrouvant autour de représentations communes, les villégiateurs ont réagi en mettant l'accent sur la spécificité et l'originalité de leur pratique. Parce que remise en cause par un acteur extérieur l'identité du groupe en est renforcée. L'État joue alors le rôle d'un «ennemi» commun, entraînant une forte cohésion du groupe en mettant entre parenthèse, au moins le temps du conflit, les éventuelles divergences internes.

\subsection{Opposition et compromis : vers une évolution des représentations sociales?}

L'opposition entre représentants de l'État et villégiateurs a connu plusieurs rebondissements. La première période prend la forme d'un conflit ouvert, avec d'une part des procédures judiciaires, et des enrochements mis en œuvre par l'État, d'autre part des manifestations et des mobilisations organisées par les villégiateurs, associations en tête. En un second temps, le conflit prend une tournure plus nuancée. L'enrochement, entamé sur Piémanson par exemple, reste inachevé, permettant encore le passage des véhicules.

Il s'agit là d'une situation intermédiaire ne proposant pas de solutions définitives. Cette nuanciation du conflit serait révélatrice de l'évolution des représentations du site, et plus largement de la nature, propres aux deux antagonistes. Ainsi, les villégiateurs ont repris les arguments de l'État (hygiène, sécurité, environnement); les intégrant dans leur pratique et leur représentation du site. Ils se présentent ainsi comme de véritables gestionnaires, associant à leur auto-organisation ces préoccupations hygiénistes, sécuritaires et environnementales. Ils proposent une amélioration de leur équipement sanitaire (W.-C. chimiques, fosses septiques), montrent leur capacité à respecter des règles de sécurité en particulier pour la vitesse de circulation et s'impliquent dans des actions de protection de l'environnement, comme les grands nettoyages de printemps et de fin de saison estivale, ainsi que la plantation d'arbres et de végétaux pour le maintien des dunes. De son côté, l'État opte pour des démarches moins radicales, retardant l'interdiction totale de la villégiature.

À Beauduc, cela s'est traduit par l'élaboration d'une charte, véritable cahier des charges, visant à proposer une réglementation du site et des solutions aux problèmes de circulation, d'hygiène, de sécurité et d'environnement. Face à cette structuration notable du mouvement de protestation beauducois, l'État décide de diligenter une mission interministérielle chargée d'étudier les possibilités d'avenir du site et le maintien de certains usages et occupations qui s'y rattachent.

Le processus du conflit semble ainsi aboutir désormais à l'étape du compromis dont G. SimMEL (1995) dit qu'il est «une des plus grandes inventions de l'humanité». Il est en même temps révélateur d'une forme de socialisation pour ces groupes d'usagers, et d'une régulation sociale intégrant des normes et des pratiques perçues dans un premier temps comme déviantes. Celles-ci seraient en voie d'être redéfinies comme symbole d'un patrimoine culturel et naturel. Le médiateur principal dans cette étape du compromis doit une large part au thème de la sensibilité à l'environnement, d'abord source d'antagonisme puis de rapprochement, devenant une base d'unité et de consensus entre les adversaires dans ce conflit. 


\section{CONCLUSION}

Les exemples de Piémanson et de Beauduc permettraient de montrer l'évolution de l'opposition entre les couches populaires et les couches moyennes, observées au cours des années 1980 (PICON, 1987 ; Chamboredon, 1985), concernant les représentations et les pratiques de nature. Les conflits d'usage et de représentations analysés dans cette étude ne peuvent pas être résumés à l'affrontement, tout symbolique qu'il soit, entre deux groupes sociaux totalement hermétiques l'un à l'autre. Les représentations circulent non seulement au sein de chacun des groupes mais aussi entre les groupes ${ }^{17}$. La nature, lieu de prélèvement pour les couches populaires, devient aussi pour ces derniers un espace à préserver. De même, l'actuel discours véhiculé par les pouvoirs publics ne limite plus l'environnement au seul écosystème, prenant progressivement en compte l'occupation humaine des sites en question. Ces évolutions permettraient une ouverture vers de possibles compromis provisoires ou plus durables.

\section{BIBLIOGRAPHIE}

Aspe C., (1991).- Une histoire entre les couches moyennes et la localité, in Chercheurs d'eau en Méditerranée, ed. du Félin, Paris.

Becker H., (1985).- Outsiders, ed. A.M. Métailé, Paris.

Chamboredon J.C., (1985). - La naturalisation de la campagne : une autre manière de cultiver les simples?, in Protection de la nature, histoire et idéologie: de la nature à l'environnement, CADORET A. (dir.), L'Harmattan, Paris.

Chazel F., (1997).- Normes et valeurs sociales, Encyclopaedia Universalis.

CoRBIn A., (1988).- Le territoire du vide. L'Occident et le désir du rivage 1750-1840. Aubier, Paris.

Corbin A., (1995).- L'avènement des loisirs, 1850-1960, Aubier, Paris.

Corbin A., (1982).- Le miasme et la jonquille, Flammarion, Paris.

Doise W. \& Palmonari A., (1986).- L'étude des représentations sociales, ed. Delachaux \& Niestlé, Genève.

Dumazedier J., (1988).- Révolution culturelle du temps libre 1968-1988, Méridiens, Paris.

FABIANı J.-L., (1985).- Sciences des écosystèmes et protection de la nature, in Protection de la nature, histoire et idéologie : de la nature à l'environnement, CAdoret A. (dir.), L'Harmattan, Paris.

FleURY M.-C., (1992).- Le littoral camarguais. Inventaire des aménagements, évolution géographique, économique et juridique entre 1970 et 1992, DESS Université de Montpellier.

Jodelet D., (1989).- Les représentations sociales, PUF, Paris.

KAlAORA B., (1993).- Le musée vert, radiographie du loisir en forêt, L'Harmattan, Paris.

Picon B., (1987).- Les conflits d'usage sur le littoral camarguais, Norois, tome 34, $\mathrm{n}^{\circ} 133-135$.

Picon B., (1985).- La protection de la nature sur le littoral camarguais : du tourisme de masse à la fréquentation élitiste, Anthropologie Maritime, cahier $n^{\circ} 2$.

Picon B., (1988).- L'espace et le temps en Camargue, Actes Sud, Arles.

Simmel G., (1990).- Philosophie de la modernité, tome II, Payot, Paris.

Simmel G., (1995).- Le conflit, Circée, Dijon.

Urbain J.D., (1994).- Sur la plage, mours et coutumes balnéaires, Payot, Paris.

\section{Notes}

1 - Le corps de métiers des baliseurs avait été créé dès le milieu du XVII ${ }^{e}$ siècle, afin de faciliter la navigation particulièrement difficile le long de cette côte instable et dangereuse, les agents de la santé étaient chargés quant à eux, de surveiller l'état sanitaire des bâtiments marins, de leur équipage et de leur chargement afin d'éviter la contagion d'épidémies avec l'intérieur du pays.

2- Ils se servent alors des voiles de leurs bateaux comme campement sommaire.

3- Ouvrage du milieu du XIXe siècle.

4-Institutes de Justinien, livre II, titre 1, § 3, 555 après J.-C.

5- Portion d'estran régulièrement recouvert par la mer.

6- Tellines, palourdes, clovisses, couteaux, coques...

7- 80 pêcheurs professionnels exercent leur activité sur l'ensemble du littoral camarguais (plage des SaintesMaries, golfe de Beauduc et plage de Piémanson). Il s'agit de pêcheurs de tellines.

8- Ce phénomène avait aussi été observé sur la plage des Saintes-Maries, lors d'une enquête similaire (PICON, 1985, 1987)

9- Concernant l'enquête quantitative auprès des usagers de la plage de Piémanson $76 \%$ des ouvriers rencontrés sont des campeurs contre seulement $35 \%$ des cadres.

10- On compte par exemple jusqu'à 15 constructions par an entre 1960 et 1967.

11- Certains Beauducois ont construit leur habitat en fonction d'un réseau de parenté ou d'alliance, on a ainsi des familles regroupées autour de plusieurs cabanons et/ ou caravanes, on a également des alliances basées sur la vicinalité et qui font de certains cabaniers ou caravaniers des voisins à la ville comme à la mer.

12- On entend par représentation sociale une forme de connaissance socialement et culturellement construite 
(Doise \& Palmonari, 1986 ; Jodelet, 1989).

13- Nous retiendrons comme définition de la norme celle proposée par F. Chazel (1997): «dans le langage sociologique, une norme constitue une règle ou un critère régissant notre conduite en société. Il ne s'agit pas d'une régularité statistique dans les comportements observés, mais d'un modèle culturel de conduite auquel nous sommes censés nous conformer».

14- Un événement marquant de cette préoccupation étatique est la création du ministère de l'Environnement en 1971. Concernant le littoral, la loi Littoral de 1986 accorde une place considérable à la protection de la nature. Ces événements marquant sont le fruit d'une évolution de la politique de l'État concernant la gestion et la protection de la nature, amorcée à la fin du XIX ${ }^{e}$ siècle par le corps forestier étatique (KALAORA, et SAVOYE, 1985 ; LarRère et Poupardin, 1985).

15- Ces couches sociales culturellement dominantes sont désignées par les sociologues comme «couches moyennes intellectuelles» (PICON, 1988 ; CHAMBOREDON, 1985 ; ASPE, 1991).

16- G. Simmel (1990) analyse ce processus, montrant la «valeur constructive de l'opposition».

17- Ce résultat renvoie à l'analyse de G. Simmel (1990), montrant que le conflit est une forme de socialisation et que «le combat est déjà la solution de la division entre les contraires». 